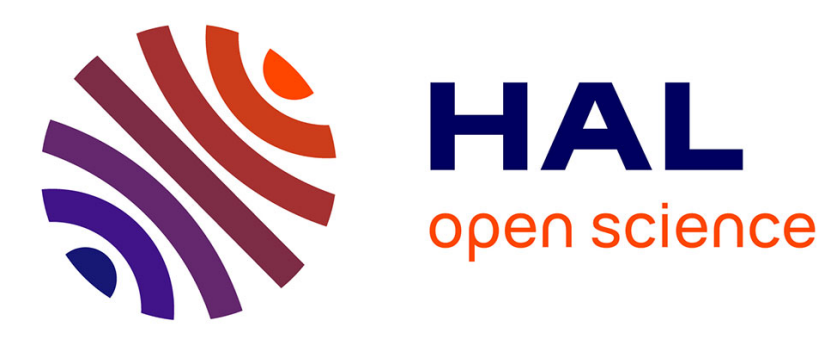

\title{
Power Allocation Optimization in NOMA-Based Multi-Cell VLC Networks
}

Mahmoud Wafik Eltokhey, Mohammad-Ali Khalighi, Zabih Ghassemlooy

\section{To cite this version:}

Mahmoud Wafik Eltokhey, Mohammad-Ali Khalighi, Zabih Ghassemlooy. Power Allocation Optimization in NOMA-Based Multi-Cell VLC Networks. 2021 17th International Symposium on Wireless Communication Systems (ISWCS), Sep 2021, Berlin, Germany. pp.1-5, 10.1109/ISWCS49558.2021.9562144 . hal-03452594

\section{HAL Id: hal-03452594 \\ https://hal.science/hal-03452594}

Submitted on 27 Nov 2021

HAL is a multi-disciplinary open access archive for the deposit and dissemination of scientific research documents, whether they are published or not. The documents may come from teaching and research institutions in France or abroad, or from public or private research centers.
L'archive ouverte pluridisciplinaire HAL, est destinée au dépôt et à la diffusion de documents scientifiques de niveau recherche, publiés ou non, émanant des établissements d'enseignement et de recherche français ou étrangers, des laboratoires publics ou privés. 


\section{Power Allocation Optimization in NOMA-Based Multi-Cell VLC Networks}

\author{
$1^{\text {st }}$ Mahmoud Wafik Eltokhey \\ Aix-Marseille University, CNRS, \\ Centrale Marseille, Institut Fresnel \\ Marseille, France \\ Mahmoud.Eltokhey@centrale-marseille.fr
}

\author{
$2^{\text {nd }}$ Mohammad-Ali Khalighi \\ Aix-Marseille University, CNRS, \\ Centrale Marseille, Institut Fresnel \\ Marseille, France \\ Ali.Khalighi@fresnel.fr
}

\author{
$3^{\text {rd }}$ Zabih Ghassemlooy \\ Optical Communications Research Group \\ Northumbria University \\ Newcastle upon Tyne NE1 8ST, UK \\ Z.Ghassemlooy@northumbria.ac.uk
}

\begin{abstract}
The use of visible-light communication (VLC) to complement radio-frequency systems in satisfying the everincreasing user data demands has received particular interest since about two decades. One important issue in VLC networks is to address the multiple-access (MA) requirement. Non-orthogonal MA (NOMA) is a promising technique for multi-cell VLC networks, in particular, due to providing high spectral efficiency. However, its performance is highly impacted by the considered power allocation (PA). In this paper, we propose optimization of PA in NOMA-based VLC networks using particle swarm optimization. The presented results show that the optimized PA offers a higher performance in terms of the network achievable throughput and fairness, compared with non-optimized PA, while offering flexibility in controlling the desired performance criteria.
\end{abstract}

Index Terms-Visible-light communications; Multiple-access techniques; NOMA; Particle swarm optimization.

\section{INTRODUCTION}

Visible-light communication (VLC) has been receiving an increasing interest for supporting indoor and outdoor wireless access, due to several features including: the presence of unregulated spectrum, immunity against the radio-frequency (RF) interference, inherent physical layer security, and the reliance on using light-emitting diode (LED)-based lighting infrastructures [1]. To support lighting and data communication in relatively large space scenarios, multiple LED luminaires are used, each one serving as an access point (AP), providing service to the users within its coverage area. In such a multicell VLC network, according to the locations of the users with respect to the APs, users can be classified as either of cell-center users (CCUs) or cell-edge users (CEUs). The former refers to the users within the coverage area of only one AP, while the latter are the users located within the coverage areas of more than one AP. In such networks, the users performances are affected by the inter-cell interference (ICI), resulting from received signals from neighbouring cells, and inter-user interference (IUI) due to the received signals of other users in the same cell.

One efficient multiple-access (MA) technique to minimize IUI and ICI effects is the power-domain NOMA (simply referred to as NOMA here), which exploits the differences in the channel gain between users to allow multiplexing their signals [2]. At the receiver $(\mathrm{Rx})$ side, successive interference cancellation (SIC) is done to separate the signals of different users [3]. Several works have compared the performances of orthogonal frequency-division MA (OFDMA) and NOMA in single-cell and multi-cell scenarios, where the advantage of the latter scheme was shown in terms of network throughput, especially for increased number of users [3]-[5].

Several works proposed solutions for improving the sumrate in single-cell NOMA-VLC networks. In [6]-[8], power allocation (PA) schemes were proposed, which outperformed the gain ratio PA. Cuckoo Search (CS) optimization was used in [9] to optimize the VLC system parameters, including PA, with the objective of maximizing both the received power and the achievable rate. In [10], a joint power-line communication (PLC)-VLC PA strategy was proposed to maximize the network sum-rate. Optimal user pairing followed by PA using majorization minimization was considered in [11] to maximize the sum-rate for VLC with steerable beams.

In contrast, fewer works have investigated IUI and ICI management in multi-cell NOMA-VLC networks. In [12], overlapped clustering based on hybrid NOMA- orthogonal multiple access (OMA) were considered in layered asymmetricallyclipped optical orthogonal-frequency division multiplexing (LACO-OFDM) VLC networks, for minimizing interference. User assignment was considered in [13] for ICI mitigation, where PA was optimized using the gradient projection algorithm for sum-rate maximization. In [14], ICI mitigation was carried out by using zero-forcing pre-coding for broadcasting the user signals as the highest decoding order NOMA user, while [15] coordinated the scheduling of NOMA transmission in each cell to mitigate ICI, while satisfying the dimming requirements in the network.

In this paper, we propose the optimization of PA in multicell NOMA-VLC networks using particle swarm optimization (PSO), which has been used in solving different problems in optical communications [16]-[18]. By tuning the PA in each of the cells, both IUI and ICI in the network can be controlled, while by tuning the optimization parameters, the desired performance could be improved, considering the trade-off between network throughput maximization and the homogeneity of user performance (i.e., fairness). The results show improved performance compared with non-optimized PA in terms of both network throughput and fairness. 


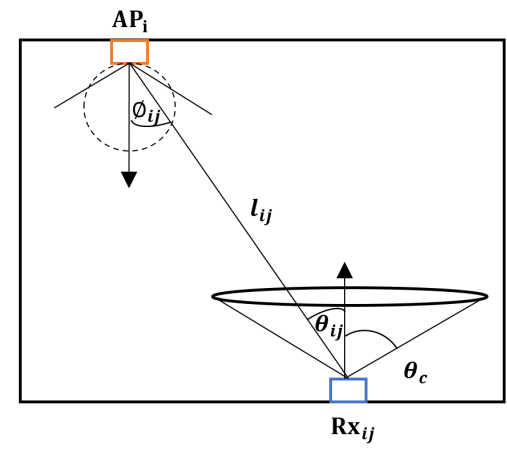

Fig. 1: Illustration of the link parameters.

The remainder of the paper is organized as follows: Section II presents the system model, Section III outlines a brief analysis of NOMA signalling. Section III presents the proposed PSO-based PA optimization, and Section V discusses the results of comparing the optimized and non-optimized PA. Finally, Section VI concludes the paper.

\section{SySTEM MOdEL}

We consider an intensity modulation/direct detection multicell VLC network, that has $N_{t}$ APs (LED luminaires) handling a total number of $N_{r}$ users, such that each $\mathrm{AP}_{i}$ handles $N_{i}$ users, and the channel gain for the link between $\mathrm{AP}_{i}$ and the user $\mathrm{Rx}_{i j}$ is denoted by $h_{i j}$. Assuming Lambertian pattern for the LED transmission of order $O$ and that the line-of-sight component dominates the channel gain, we have [19]:

$$
h_{i j}=\mathcal{S} \frac{(O+1) \mathcal{R} A}{2 \pi \ell_{i j}^{2}} \cos ^{O}\left(\phi_{i j}\right) \cos \left(\theta_{i j}\right),
$$

where $\mathcal{S}$ refers to the LED conversion efficiency, $\phi_{i j}$ is the transmission angle at the $\mathrm{AP}_{i}, \theta_{i j}$ is the angle of incidence at the $\mathrm{Rx}_{i j}, \mathcal{R}$ is the responsivity of the photo-detector (PD), and $\ell_{i j}$ is the path length, see Fig. 1. Also, denoting the active area of the PD, the Rx field-of-view (FOV), and the optical concentrator's refractive index by $A_{\mathrm{PD}}, \theta_{c}$, and $q$, respectively, the Rx collection area $A$ is given by:

$$
A=\frac{q^{2}}{\sin ^{2}\left(\theta_{c}\right)} A_{\mathrm{PD}}
$$

\section{NOMA SIGNALING}

Considering $N_{t}$ APs, each one handling $N_{i}$ users, $i=$ $1, \ldots, N_{t}$, the users' signals are multiplexed by the AP in power domain using superposition coding. For this, users' signals are assigned different power levels, where the users with the lowest channel gains are assigned the highest power levels, and have the lowest decoding order. At the users side, SIC detection is carried out, where users with higher decoding order decode the signals of the users with lower decoding order before accessing their signals. Assuming availability of
CSI at all Rxs, the signal received by $\mathrm{Rx}_{i j}$ handled by $\mathrm{AP}_{i}$ is given by:

$$
\begin{aligned}
r_{i j}= & a_{i j} \sqrt{P_{e}} h_{i j} d_{i j}+\sum_{k=1}^{j-1} a_{i k} \sqrt{P_{e}} h_{i j} d_{i k} \\
& +\sum_{k=j+1}^{N_{i}} a_{i k} \sqrt{P_{e}} h_{i j} d_{i k}+z_{j},
\end{aligned}
$$

where $P_{e}$ refers to the total electrical transmit power of the $\mathrm{AP}_{i}, a_{i j}$ represents the PA weight, $d_{i j}$ denotes the desired data of $\mathrm{Rx}_{i j}$, and $z_{j}$ refers to Gaussian noise with variance $\sigma_{n}^{2}$. In (3), the first, second, and third terms represent the desired signal, the interference components cancelled by SIC, and residual interference following SIC, respectively. By assuming static PA for sake of simplicity, we have $a_{i j}^{2}=\alpha a_{i j-1}^{2}$, such that $0<\alpha<1$ refers to the PA coefficient, which decides the ratio between the power allocated to a user and the preceding user in the decoding order. Note that, $\sum_{j=1}^{N_{i}} a_{i j}^{2}=1$ for ensuring normalized electrical transmit power. The signalto-interference-plus-noise ratio (SINR) for the $\mathrm{Rx}_{i j}$ is then calculated as:

$$
\operatorname{SINR}_{\text {NOMA }, \mathrm{Rx}_{i j}}=\frac{h_{i j}^{2} P_{e} a_{i j}^{2}}{I_{\mathrm{ICI}}+h_{i j}^{2} P_{e} \sum_{k>j} a_{i k}^{2}+\sigma_{n}^{2}},
$$

where $I_{\text {ICI }}$ refers to the ICI component. The maximum achievable throughput of $\mathrm{Rx}_{i j}$ is then given by:

$$
R_{i j}=\frac{B}{2} \log _{2}\left(1+\operatorname{SINR}_{\mathrm{NOMA}, U_{i j}}\right) \quad \text { (bps), }
$$

where $B$ denotes the system bandwidth, and the loss factor of 2 is due to the Hermitian symmetry constraint, assuming DCO-OFDM signalling.

To measure the homogeneity of users' throughput performance, we consider Jain's fairness index, defined as [15], [20]:

$$
\mathrm{FI}=\frac{1}{N_{r}} \frac{\left(\sum_{i=1}^{N_{t}} \sum_{j=1}^{N_{i}} R_{i j}\right)^{2}}{\sum_{i=1}^{N_{t}} \sum_{j=1}^{N_{i}} R_{i j}^{2}} .
$$

FI $=1$ in case that all $R_{i j}$ are equal; it decreases with increase in the difference between the users achievable throughputs.

\section{PA Optimization Using Particle Swarm OPTIMIZATION}

For optimizing the PA coefficients to improve the network performance, we consider the use of PSO algorithm [21]. In PSO, particles move in the solution space to find their optimal solution, in a way similar to the behavior of swarms of bees searching for locations with highest densities of flowers [21], [22].

The movements of each of the particles in PSO are influenced by the best position reached by the particle itself so far, which is referred to as personal best position Pbest, and 
by the global best position Gbest reached by all particles. For evaluating the quality of the solution according to the desired performance criteria, a fitness function is considered. For the number of iterations $N_{i t}$ and of particles $N_{p}$ used in $D$ dimensions optimization problem (i.e., $D$ optimized variables), at iteration $l$, the PSO algorithm updates, for the particle $m$, the vectors of position $\boldsymbol{p}_{m}=\left[p_{m 1}, p_{m 2}, \ldots, p_{m D}\right]$; velocity $\boldsymbol{v}_{m}=$ $\left[v_{m 1}, v_{m 2}, \ldots, v_{m D}\right]$; and personal best position $\boldsymbol{P b e s t}_{\boldsymbol{m}}=$ $\left[\right.$ pbest $_{m 1}$, pbest $_{m 2}, \ldots$, pbest $\left._{m D}\right]$. In case that the $m^{\text {th }}$ particle performance is better than that of the global best position vector Gbestv $=\left[\right.$ gbest $_{1}$, gbest $_{2}, \ldots$, gbest $\left._{D}\right]$, the position of the particle is used as the Gbestv. The velocity of the $m^{\text {th }}$ particle at iteration $l$ for the variable $d$ is then given by [21]:

$$
\begin{aligned}
v_{m d}^{l+1}= & w^{l} v_{m d}^{l}+c_{P}^{l} \operatorname{rand}_{P}\left(\text { pbest }_{m d}-p_{m d}^{l}\right) \\
& +c_{G}^{l} \operatorname{rand}_{G}\left(\text { gbest }_{d}-p_{m d}^{l}\right),
\end{aligned}
$$

where the first, second, and third terms refer to the contributions from the components of the particle's old velocity, and the vectors of Pbest and Gbest, respectively. $w^{l}$ denotes the inertia weight that controls the contribution of the old value of the velocity on calculation of its new value, and $\operatorname{rand}_{P}$ and $\operatorname{rand}_{G}$ refer to random numbers bounded between 0 and $1 . c_{P}^{\ell}$ and $c_{G}^{\ell}$ correspond to the weights that control the contributions of Pbest and Gbest vectors on determining the new velocity. Considering the time step $\Delta t$, the new position of the $m^{\text {th }}$ particle at iteration $l+1$ for the variable $d$ is:

$$
p_{m d}^{l+1}=p_{m d}^{l}+v_{m d}^{l+1} \Delta t
$$

For optimizing PA coefficients, in order to highlight the role of the fitness function in controlling the network performance, three different fitness functions are considered. The first fitness function focuses only on maximizing the sum-rate:

$$
F_{S R}=\sum_{i=1}^{N_{t}} \sum_{j=1}^{N_{i}} R_{i j}
$$

which promotes solutions resulting in sum-rate improvement. The second fitness function maximizes both sum-rate and FI:

$$
F_{S R+F I}=\sum_{i=1}^{N_{t}} \sum_{j=1}^{N_{i}} R_{i j}+W_{1} \mathrm{FI},
$$

where the first and second terms promote solutions improving the sum-rate and FI, respectively. The weight $W_{1}$ ensures comparable contributions of the two terms. Assuming the sumrate on the order of $10 \mathrm{Mbps}$ and given the maximum FI of $1, W_{1}$ is set to $10^{8}$ here. The third considered fitness function focuses only on maximizing the FI:

$$
F_{F I}=W_{2} \mathrm{FI}
$$

where $W_{2}$ is set to $10^{9}$. Figure 2 shows the flow chart for the proposed optimization algorithm. It highlights generating random positions, evaluation of the positions (PA coefficients), and the update of the new positions to be explored, for finding the optimal solution (i.e., the final global best position).

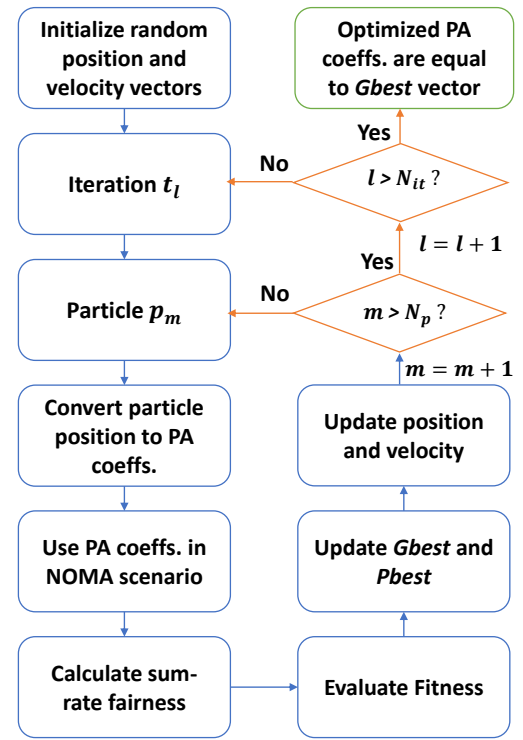

Fig. 2: Flow chart of the proposed PA optimization algorithm. Parameters $l$ and $m$ denote the indexes of numbers of iterations and particles, respectively.

TABLE I: Simulation parameters [23], [24]

\begin{tabular}{cc}
\hline Parameter & Value \\
\hline Room dimension & $\left(7 \times 7 \times 3 \mathrm{~m}^{3}\right)$ \\
LED Lamertian order $O$ & 1 \\
Number of LED chips per LED luminaire & 36 \\
Signal current per LED chip & $100 \mathrm{~mA}$ \\
LED conversion efficiency $\mathcal{S}$ & $0.44 \mathrm{~W} / \mathrm{A}$ \\
PD area & $1 \mathrm{~cm}^{2}$ \\
Refractive index of the optical concentrator & 1.5 \\
Rx's FOV & $62 \mathrm{deg}$. \\
PD responsivity $\mathcal{R}$ & $0.4 \mathrm{~A} / \mathrm{W}$ \\
System bandwidth $B$ & $10 \mathrm{MHz}$ \\
Equivalent Rx noise power spectral density & $10^{-21} \mathrm{~A}^{2} / \mathrm{Hz}$ \\
\hline
\end{tabular}

\section{PERformance Study}

We compare here the performances of the non-optimized PA and the proposed optimized PA over different scenarios.

\section{A. Main Assumptions and Considered Scenarios}

We consider three different 4-cell VLC network scenarios, with different combinations of CCUs and CEUs. In Scenario 1, $N_{r}=8$ and the number of CEUs is set to 4; in Scenario 2, $N_{r}=12$ including 4 CEUs; and in Scenario 3, $N_{r}=16$ including 8 CEUs. The APs are positioned at the cell centers with the transmit optical power $P_{o}=1.584 \mathrm{~W}$ [23]. Also, the heights of the APs and the Rxs from the floor level are set to 2.5 and $0.85 \mathrm{~m}$, respectively. We consider $N_{p}=N_{i t}=10$ as appropriate parameters for PSO. For each scenario, 200 random user positions are generated to determine the average sum-rate and fairness performance. ${ }^{1}$ Table I summarizes the simulation parameters. Note that, the PA coefficients in any cell are bounded between 0.1 and 0.5 . Also, for the cases of

\footnotetext{
${ }^{1}$ Note that in the simulations we exclude those scenarios resulting in CEUs located in coverage areas of more than two APs for simplicity. However, the proposed optimization could be applied to such cases in a future work.
} 
non-optimized PA, we consider PA coefficients of $0.1,0.2,0.3$, 0.4 , and 0.5. The PSO parameters $w^{\ell}, c_{P}^{l}$, and $c_{G}^{l}$ are varied according to [17], [22], [25] to ensure a quick conversion of the algorithm.

\section{B. Numerical Results}

Figure 3 contrasts the average sum-rate and fairness performances over the 200 random user positions in Scenarios 1, 2, and 3 for the cases of non-optimized and the optimized PA.

Consider first the case of non-optimized PA coefficients, where the results show that increasing the PA coefficient results in increased network sum-rate and decreased FI. This results from the more opportunistic approach adopted by the network. Indeed, increasing the PA coefficients results in allocating higher power levels for the users with higher decoding orders (having higher channel gains). This results in maximized network sum-rate but in decreasing the power allocated to the users with lower decoding order, thus penalizing them. Consequently, the margin between the achievable throughputs of users is increased, thus the fairness performance.

We notice an improvement in the sum-rate performance when moving from Scenario 1 to Scenario 2, due to handling of more users. Also, we notice a degraded sum-rate performance by moving from Scenario 2 to Scenario 3, due to the increase in the IUI experienced by the users (see (4)).

Generally, the results show the merits of adopting optimized versus non-optimized PA coefficients. In particular, by adopting $F_{S R}$ and $F_{S R+F I}$ fitness functions, an improved sumrate performance is achieved for all considered values of nonoptimized PA. On the other hand, adopting $F_{F I}$ results in an improved FI performance, compared with the cases with non-optimized PA. Note that, by moving from the fitness functions $F_{S R}$ to $F_{S R+F I}$ and then $F_{F I}$, the sum-rate performance degrades, while the fairness performance improves. This was expected, since $F_{S R}$ considers only the sum-rate, thus resulting in the best sum-rate and the worst fairness performances. On the other hand, $F_{F I}$ promotes only the fairness performance, thus resulting in the best FI performance and the lowest sum-rate. The $F_{S R+F I}$ fitness function offers a tradeoff between sum-rate and fairness, compared to the two other ones. The results show that, by tuning the fitness function, the desired performance criterion could be improved. This could be considered as a degree of freedom in adapting NOMA scheme to the changing network conditions or requirements. Note that, the space and time complexities of the considered PSO algorithm are given by $O\left(N_{p} \times D\right)$ and $O\left(N_{i t} \times N_{p} \times D\right)$, respectively [17].

\section{CONClusions}

We proposed PSO-based optimization of PA coefficients in NOMA-based multi-cell VLC networks using different fitness functions, and compared the achieved performance with the non-optimized PA NOMA case. Our results showed improved performance in terms of sum-rate and fairness. In particular, tuning the fitness function provides the flexibility to adapt to the changing network requirements or criteria. The achieved

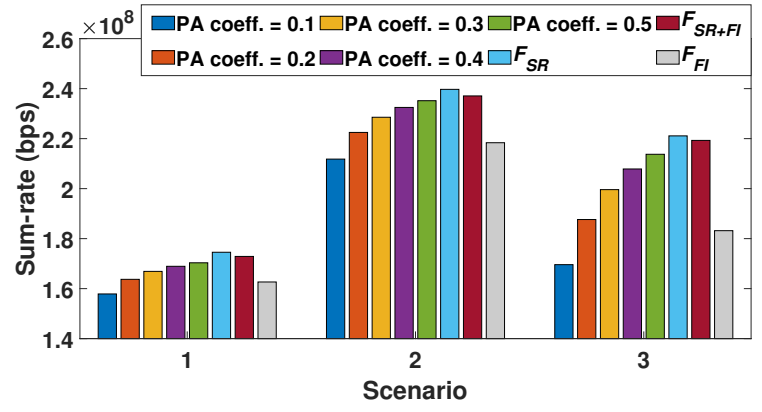

(a) Sum-rate

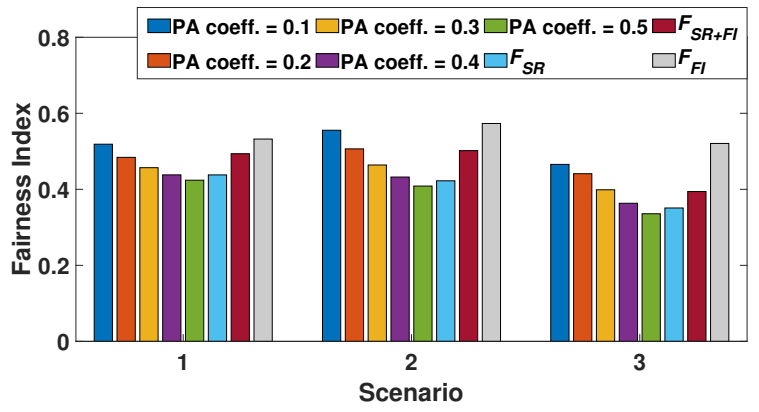

(b) FI

Fig. 3: Comparison of (a) sum-rate and (b) fairness index for the different considered optimization fitness functions and different values for non-optimized PA coefficients.

performance improvement by optimized PA comes at the cost of increased network complexity, which remains rather acceptable, given the relatively small number of evaluations needed for carrying out the optimization. Future work will consider applying the proposed optimization to other PA techniques (such as gain ratio PA), and including other system parameters in the optimization (e.g., user association for CEUs).

\section{ACKNOWLEDGMENT}

This work has received funding from the European Union's Horizon 2020 research and innovation programme under the Marie Skłodowska-Curie grant agreement No. 764461 (VisIoN), and was also based upon work from European Union's Horizon 2020 COST Action CA19111 (NEWFOCUS).

\section{REFERENCES}

[1] Z. Ghassemlooy, L. N. Alves, S. Zvanovec, and M. A. Khalighi, Eds., Visible Light Communications: Theory and Applications. CRC-Press, 2017.

[2] O. Maraqa, A. S. Rajasekaran, S. Al-Ahmadi, H. Yanikomeroglu, and S. M. Sait, "A survey of rate-optimal power domain NOMA with enabling technologies of future wireless networks," IEEE Communications Surveys Tutorials, vol. 22, no. 4, pp. 2192-2235, 2020.

[3] R. C. Kizilirmak, C. R. Rowell, and M. Uysal, "Non-orthogonal multiple access (NOMA) for indoor visible light communications," in International Workshop on Optical Wireless Communications (IWOW), Sept. 2015, pp. 98-101, Istanbul, Turkey.

[4] L. Yin, W. O. Popoola, X. Wu, and H. Haas, "Performance evaluation of non-orthogonal multiple access in visible light communication," IEEE Transactions on Communications, vol. 64, no. 12, pp. 5162-5175, Dec. 2016. 
[5] M. W. Eltokhey, M. A. Khalighi, and Z. Ghassemlooy, "Multiple access techniques for large space indoor scenarios: A comparative study,," in International Conference on Telecommunications (ConTEL), July 2019, Graz, Austria.

[6] Z. Yang, W. Xu, and Y. Li, "Fair non-orthogonal multiple access for visible light communication downlinks," IEEE Wireless Communications Letters, vol. 6, no. 1, pp. 66-69, Feb. 2017.

[7] Y. Fu, Y. Hong, L. Chen, and C. W. Sung, "Enhanced power allocation for sum rate maximization in OFDM-NOMA VLC systems," IEEE Photonics Technology Letters, vol. 30, no. 13, pp. 1218-1221, Jul. 2018.

[8] Q. Li, T. Shang, T. Tang, and Z. Dong, "Optimal power allocation scheme based on multi-factor control in indoor NOMA-VLC systems," IEEE Access, vol. 7, pp. 82 878-82 887, 2019.

[9] Z. Tahira, H. M. Asif, A. A. Khan, S. Baig, S. Mumtaz, and S. AlRubaye, "Optimization of non-orthogonal multiple access based visible light communication systems," IEEE Communications Letters, vol. 23 , no. 8, pp. 1365-1368, 2019.

[10] S. Feng, T. Bai, and L. Hanzo, "Joint power allocation for the multi-user NOMA-downlink in a power-line-fed VLC network," IEEE Transactions on Vehicular Technology, vol. 68, no. 5, pp. 5185-5190, 2019.

[11] Y. S. Eroglu, C. K. Anjinappa, . Guvenc, and N. Pala, "Slow beam steering and NOMA for indoor multi-user visible light communications," IEEE Transactions on Mobile Computing, vol. 20, no. 4, pp. 1627-1641, 2021.

[12] S. Feng, R. Zhang, W. Xu, and L. Hanzo, "Multiple access design for ultra-dense VLC networks: Orthogonal vs non-orthogonal," IEEE Transactions on Communications, vol. 67, no. 3, pp. 2218-2232, Mar. 2019.

[13] X. Zhang, Q. Gao, C. Gong, and Z. Xu, "User grouping and power allocation for NOMA visible light communication multi-cell networks," IEEE Communications Letters, vol. 21, no. 4, pp. 777-780, Apr. 2017.

[14] M. W. Eltokhey, M. A. Khalighi, A. S. Ghazy, and S. Hranilovic, "Hybrid NOMA and ZF pre-coding transmission for multi-cell VLC networks," IEEE Open Journal of the Communications Society, vol. 1, pp. 513-526, 2020.

[15] M. W. Eltokhey, M. A. Khalighi, and Z. Ghassemlooy, "Dimming-aware interference mitigation for NOMA-based multi-cell VLC networks,"
IEEE Communications Letters, vol. 24, no. 11, pp. 2541-2545, 2020.

[16] M. S. Demir, S. M. Sait, and M. Uysal, "Unified resource allocation and mobility management technique using particle swarm optimization for VLC networks," IEEE Photonics Journal, vol. 10, no. 6, pp. 1-9, 2018.

[17] M. W. Eltokhey, K. R. Mahmoud, Z. Ghassemlooy, and S. S. Obayya, "Optimization of intensities and locations of diffuse spots in indoor optical wireless communications," Optical Switching and Networking, vol. 33, pp. $177-183,2019$.

[18] M. W. Eltokhey, M. A. Khalighi, and Z. Ghassemlooy, "Optimization of receivers field-of-views in multi-user VLC networks: A bio-inspired approach," IEEE Wireless Communications, under revision.

[19] S. Long, M. A. Khalighi, M. Wolf, S. Bourenanne, and Z. Ghassemlooy, "Investigating channel frequency selectivity in indoor visible light communication systems," IET Optoelectronics, vol. 10, no. 3, pp. 80-88, May 2016.

[20] R. K. Jain, D. M. W. Chiu, and W. R. Hawe, "A quantitative measure of fairness and discrimination," Eastern Research Laboratory, Digital Equipment Corporation, Hudson, MA, 1984.

[21] J. Kennedy and R. Eberhart, "Particle swarm optimization," in Proceedings of ICNN'95 - International Conference on Neural Networks, vol. 4, Nov. 1995, pp. 1942-1948 vol.4, Perth, Australia.

[22] Y. Shi and R. Eberhart, "A modified particle swarm optimizer," in 1998 IEEE International Conference on Evolutionary Computation Proceedings. IEEE World Congress on Computational Intelligence, May 1998, pp. 69-73, Anchorage, USA.

[23] H. Ma, L. Lampe, and S. Hranilovic, "Coordinated broadcasting for multiuser indoor visible light communication systems," IEEE Transactions on Communications, vol. 63, no. 9, pp. 3313-3324, Sept. 2015.

[24] Z. Yu, R. J. Baxley, and G. T. Zhou, "Multi-user MISO broadcasting for indoor visible light communication," in International Conference on Acoustics, Speech and Signal Processing (ICASSP), May 2013, pp. 4849-4853, Vancouver, Canada.

[25] A. Ratnaweera, S. K. Halgamuge, and H. C. Watson, "Self-organizing hierarchical particle swarm optimizer with time-varying acceleration coefficients," IEEE Transactions on Evolutionary Computation, vol. 8, no. 3, pp. 240-255, Jun. 2004. 